\title{
ADJOINT SEMIGROUP THEORY FOR A VOLTERRA INTEGRODIFFERENTIAL SYSTEM
}

\author{
BY J. A. BURNS AND T. L. HERDMAN
}

Communicated by Richard Miller, June 23, 1975

I. Introduction. In this note we announce some recent results concerning the semigroup theory for a class of linear Volterra integrodifferential systems. The system under consideration has previously been studied by Barbu and Grossman [2] and Miller [6], via semigroup methods. Although semigroup theory is employed in both of the above mentioned articles, it is important to note that the semigroup constructed in [6] differs greatly from the semigroup constructed in [2]. In particular, Miller is able to obtain certain stability results that do not hold for the semigroup constructed in [2]. However, we show that by an appropriate choice of the state space, Miller's semigroup may be considered as the "adjoint" semigroup (in the sense of Hille and Phillips [5]) to the semigroup constructed by Barbu and Grossman. We shall state the results without proofs. Proofs of the theorems will appear elsewhere (see [3]).

II. Preliminaries. If $x:(-\infty, 0] \rightarrow C^{n}$ is given, then for $t \geqslant 0$, we define $x_{t}:(-\infty, 0] \rightarrow C^{n}$ by $x_{t}(s)=x(t+s)$. For $1 \leqslant p \leqslant+\infty$, the usual Lebesgue space of $C^{n}$-valued functions on an interval with endpoints $-\infty \leqslant a<b \leqslant+\infty$ will be denoted by $L_{p}(a, b)$. Throughout this paper, $M$ shall denote an $n \times n$ constant matrix and $K(\cdot)$ shall denote an $n \times n$ matrix function satisfying $\int_{0}^{+\infty}\|K(s)\| d s<+\infty$. Consider the linear Volterra integrodifferential equation,

$$
x^{\prime}(t)=M x(t)+\int_{-\infty}^{t} K(t-s) x(s) d s,
$$

with the initial data

$$
x(0)=\eta, \quad x_{0}(s)=\varphi(s) \quad \text { a.e. on }(-\infty, 0],
$$

where $\eta \in C^{n}$ and $\varphi \in L_{1}(-\infty, 0)$.

A solution to system (2.1)-(2.2) is a function $x:(-\infty,+\infty) \rightarrow C^{n}$ such that $x$ is absolutely continuous (A.C.) on $[0,+\infty)$ and satisfies (2.1) a.e. on $[0,+\infty), x(0)=\eta$, and $x_{0}(s)=\varphi(s)$ a.e. on $(-\infty, 0]$. We shall let $Z_{1}=C^{n} \times$ $L_{1}(-\infty, 0)$ denote the product space with the product norm. It can be shown

AMS (MOS) subject classifications (1970). Primary 45A05, 45D05. group.

Key words and phrases. Volterra integrodifferential system, semigroup, adjoint semi- 
that for each pair $(\eta, \varphi) \in Z_{1}$, there is a unique solution to (2.1) with initial data (2.2). Moreover, this solution depends continuously on the initial data $(\eta, \varphi)$. For $t \geqslant 0$ define $S(t): Z_{1} \rightarrow Z_{1}$ by $S(t)(\eta, \varphi)=\left(x(t), x_{t}(\cdot)\right)$, where $x$ is the unique solution of (2.1)-(2.2). It follows that $S(t)$ is a $C_{0}$ semigroup on $Z_{1}$. (Compare this semigroup with the one constructed in [2] .)

THEOREM 1. If A denotes the infinitesimal generator of $S(t)$, then:

(i) $A$ is closed and densely defined,

(ii) $D(A)=\left\{(\eta, \varphi) \in Z_{1}: \varphi\right.$ is $A$. C. on compact subsets of $(-\infty, 0], \varphi^{\prime} \in$ $\left.L_{1}(-\infty, 0), \varphi(0)=\eta\right\}$,

(iii) if $(\eta, \varphi) \in D(A)$, then $A(\eta, \varphi)=\left(M \eta+\int_{-\infty}^{0} K(-s) \varphi(s) d s, \varphi^{\prime}\right)$.

The adjoint space $Z_{1}^{*}$ is seen to be $Z_{\infty}=C^{n} \times L_{\infty}(-\infty, 0)$. Moreover, by easy calculations we have the following result.

THEOREM 2. If $A^{*}$ denotes the adjoint of $A$, then:

(i) $D\left(A^{*}\right)=\left\{(\xi, \Psi) \in Z_{\infty}: \Psi\right.$ is $A$. C. on compact subsets of $(-\infty, 0]$, and $\left.\hat{\Psi} \in L_{\infty}(-\infty, 0)\right\}$, where $\hat{\Psi}(s)=K^{*}(-s) \xi-\Psi^{\prime}(s)$,

(ii) if $(\xi, \Psi) \in \mathcal{D}\left(A^{*}\right)$, then $A^{*}(\xi, \Psi)=\left(M^{*} \xi+\Psi(0), \hat{\Psi}\right)$,

(iii) $\mathcal{D}\left(A^{*}\right)$ is not dense in $Z_{\infty}$ and $\overline{D\left(A^{*}\right)}=Z_{1}^{+}=\{(\xi, \Psi): \Psi \in \operatorname{BUC}(-\infty, 0]\}$ where $\mathrm{BUC}(-\infty, 0]$ is the set of all bounded, uniformly continuous functions defined on $(-\infty, 0]$.

3. The adjoint space. The semigroup $S(t)$ generates a corresponding semigroup $S^{*}(t)$ on $Z_{1}^{*}$. However it is well known that $S^{*}(t)$ is not a $C_{0}$ semigroup, and in order to obtain the desired continuity properties for $S^{*}(t)$ it is necessary to restrict $S^{*}(t)$ to a proper subspace of $Z_{1}^{*}$ (see [5]). Let $S^{+}(t)$ be the restriction of $S^{*}(t)$ to $Z_{1}^{+}=\overline{D\left(A^{*}\right)}$. It is well known that $S^{+}(t)$ is a $C_{0}$ semigroup on $Z_{1}^{+}$ and the infinitesimal generator of $S^{+}(t)$ is the operator $A^{+}$defined to be $A^{*}$ restricted to $D\left(A^{+}\right)=\left\{(\xi, \Psi) \in Z_{1}^{+}: \hat{\Psi}\right.$ is in $\left.\operatorname{BUC}(-\infty, 0]\right\}$. Consequently, if $(\xi, \varphi) \in \mathcal{D}\left(A^{+}\right)$then $A^{+}(\xi, \varphi)=\left(M^{*} \xi+\Psi(0), \hat{\Psi}\right)$, where $\hat{\Psi}(s)=K^{*}(-s) \xi-$ $\Psi^{\prime}(s)$.

This explicit representation of $A^{+}$provides the corresponding representation of $S^{+}(t)$.

THEOREM 3. If $(\xi, \Psi) \in Z_{1}^{+}$, then $S^{+}(t)(\xi, \psi)=\left(y(t), y^{t}(\cdot)\right)$, where $y$ is the solution to the system

$$
y^{\prime}(t)=M^{*} y(t)+\int_{0}^{t} K^{*}(t-s) y(s) d s+\Psi(-t)
$$

with initial value

$$
y(0)=\xi,
$$

and $y^{t}(\cdot):(-\infty, 0] \rightarrow C^{n}$ is defined by $y^{t}(s)=\Psi(s-t)+\int_{0}^{t} K^{*}(t-s-v) y(v) d v$. 
REMARK 1 . We identify the space $Z_{1}^{+}$with $W=\{(\xi, \Psi): \Psi \in \operatorname{BUC}[0,+\infty)\}$ by the following identification: for each function $f:(-\infty, 0] \rightarrow C^{n}$ define $F$ : $[0,+\infty) \rightarrow C^{n}$ by $F(t)=f(-t)$, for $t \geqslant 0$. Consequently, we can identify $S^{+}(t)$ with a semigroup $U(t): W \rightarrow W$ and $A^{+}$with the generator $C$ of $U(t)$, in a natural way. In particular, if $(\xi, \Psi) \in D(C)$, then $C(\xi, \Psi)=\left(M^{*} \xi+\Psi(0), \hat{\Psi}\right)$, where $\hat{\Psi}:[0,+\infty) \rightarrow C^{n}$ is now defined by $\hat{\Psi}(t)=K^{*}(t) \xi+\Psi^{\prime}(t)$.

REMARK 2. If $F:(-\infty, 0] \rightarrow C^{n}$ is a bounded continuous function, then $L(F)=f$ is the bounded uniformly continuous function defined on $[0,+\infty)$ by

$$
[L(F)](t)=f(t)=\int_{-\infty}^{0} K^{*}(t-s) F(s) d s .
$$

Let $Y\left(K^{*}\right)$ be the subspace of $W$ given by $Y\left(K^{*}\right)=\{(\xi, f): f=L(F), \xi=F(0)$, $F$ is bounded and continuous on $(-\infty, 0]\}$, and the closure of $Y\left(K^{*}\right)$ in $W$ will be denoted by $Y$.

It is now clear that Miller's semigroup (defined on $Y$ ) is the semigroup $U(t)$ restricted to $Y$. That is, Miller's semigroup can be considered as the restriction of the "adjoint" semigroup (under the identification of $W$ with $Z_{1}^{+}$) of $S(t)$. This is a useful interpretation of $U(t)$ since we may now make certain inferences concerning the relationship between the stability of $U(t)$ and the stability of solutions of (2.1)-(2.2).

As a simple example consider the following.

CoRollary 1. Let $(\eta, \varphi) \in Z_{1}$ and $x$ be the solution of (2.1)-(2.2). If $\left\{\lambda: \operatorname{Re} \lambda \geqslant 0, \operatorname{det}\left[M^{*}-\int_{-\infty}^{0} K^{*}(-s) e^{\lambda s} d s-\lambda\right]=0\right\}$ is empty, then $\|x(t)\| \rightarrow$ 0 as $t \rightarrow+\infty$.

REMARK 3. In [7], Miller considers the system (2.1)-(2.2) in a Banach space $X$, and under certain assumptions points out the direct embedding of his semigroup in $U(t)$. Again it is easy to check that this can be considered as the adjoint semigroup of $S(t)$ where $S(t)$ is defined on $X \times L_{1}((-\infty, 0], X)$.

All of the above results are special cases of general theorems for a class of linear functional differential equations with infinite delays (see [3]). For a treatment of $S(t)$ in the case of functional differential equations with finite delays, see Borisovič and Turbabin [4], Banks and Burns [1].

\section{REFERENCES}

1. H. T. Banks and J. A. Burns, An abstract framework for approximate solutions to optimal control problems governed by hereditary systems, Proc. Internat. Conf. on Differential Equations, Academic Press, New York, 1975.

2. V. Barbu and S. I. Grossman, Asymptotic behavior of linear integrodifferential systems, Trans. Amer. Math. Soc. 173 (1972), 277-288. MR 46 \#7826.

3. J. A. Burns and T. L. Herdman, Adjoint semigroup theory for a class of functional differential equations, Siam. J. Math. Anal. (to appear).

4. Ju. G. Borisovič and A.S. Turbabin, On the Cauchy problem for linear nonhomogeneous differential equations with retarded argument, Dokl. Akad. Nauk SSSR 185 (1969), 
741-744 = Soviet Math. Dokl. 10 (1969), 401-405. MR 40 \#493.

5. E. Hille and R. S. Phillips, Functional analysis and semigroups, rev. ed., Amer.

Math. Soc. Colloq. Publ., vol. 31, Amer. Math. Soc., Providence, R. I., 1957. MR 19, 664.

6. R. K. Miller, Linear Volterra integrodifferential equations as semigroups, Funkcial.

Ekvac. 17 (1974), 39-55.

7. - Volterra integral equations in a Banach space Funkcial. Ekvac. (to appear).

DEPARTMENT OF MATHEMATICS, VIRGINIA POLYTECHNIC INSTITUTE AND STATE UNIVERSITY, BLACKSBURG, VIRGINIA 24061 